\title{
Ocorrência de iatrogenias a pacientes assistidos em Unidade de Terapia Intensiva: Uma revisão integrativa da literatura
}

\author{
Occurrence of iatrogenesis in Intensive Care Unit patients: An integrative literature review \\ Aparición de iatrogénesis en pacientes de la Unidad de Cuidados Intensivos: Una revisión \\ bibliográfica integradora
}

Bruna Karine Oliveira do Carmo ${ }^{1 *}$, Brena Pereira dos Santos ${ }^{2}$, Claudia Lima do Nascimento ${ }^{1}$, Nadja da Fonseca Veloso 3 , Nayara Diniz Pamplona', Raisa Silva Martins ${ }^{1}$, Tamyris Ayline Maia Novais .

\section{RESUMO}

Objetivo: Analisar a produção científica acerca da ocorrência de iatrogenias em pacientes assistidos em unidades de terapia intensiva, publicada em artigos nacionais de 2012 a 2017. Metodologia: Trata-se de uma revisão integrativa da literatura, realizada nas bases de dados LILACS, BDENF e SCIELO, a partir da associação dos descritores (DeCS) iatrogenia, unidade de terapia intensiva, cuidados de enfermagem, segurança do paciente e cuidados críticos. Resultado: Foram selecionados 14 artigos, que ao serem analisados, elencaram-se três categorias para discussão, sendo: iatrogenias mais comuns na Unidade de Terapia Intensiva; cuidados de enfermagem na prevenção de iatrogenias e fatores desencadeantes de iatrogenias. Conclusão: É imprescindível conhecer e entender o que são as iatrogenias, suas causas e os fatores de riscos, a fim de implementar ações efetivas para sua prevenção, evitando assim complicações desnecessárias ao paciente.

Palavras-chave: latrogenia, Unidade de Terapia Intensiva, Cuidados de Enfermagem, Segurança do Paciente, Cuidados Críticos.

\begin{abstract}
Objective: To analyze the scientific production about the occurrence of iatrogenesis in patients assisted in intensive care units, published in national articles between 2012 and 2017. Methodology: This is an integrative literature review. LILACS, BDENF and SCIELO data, from the association of the descriptors iatrogeny, intensive care unit, nursing care, patient safety, critical care. Results: The sample consisted of 14 articles, which, when analyzed, listed three categories for discussion, namely: most common Irogenogens in the ICU, Nursing care in the prevention of iatrogenesis, Triggers of latrogenic Triggers. Conclusion: It is concluded that it is essential to know and understand what are iatrogenesis, their causes and risk factors, in order to implement effective actions for their prevention, thus avoiding unnecessary complications to the patient.
\end{abstract}

Keywords: latrogeny, Intensive Care Unit, Nursing Care, Patient Safety, Critical Care.

\section{RESUMEN}

Objetivo: Analizar la producción científica sobre la aparición de la iatrogénesis en pacientes atendidos en unidades de cuidados intensivos, publicados en artículos nacionales entre 2012 y 2017. Metodología: Esta es una revisión integradora de la literatura. Datos de LILACS, BDENF y SCIELO, de la asociación de los descriptores iatrogenia, unidad de cuidados intensivos, atención de enfermería, seguridad del paciente,

\footnotetext{
1Universidade Federal do Pará. Belém-Pará. *E-mail: brunakarine bk@hotmail.com

Esamaz. Belém-Pará.

3Universidade Federal do Piauí- UFPI. Belém-Pará.
} 
atención crítica. Resultados: La muestra consistió en 14 artículos, que, cuando se analizaron, enumeraron tres categorías para la discusión, a saber: Irogenógenos más comunes en la $\mathrm{UCI}$, Atención de enfermería en la prevención de la iatrogénesis, Desencadenantes de activadores iatrogénicos. Conclusión: Se concluye que es esencial conocer y comprender qué son la iatrogénesis, sus causas y factores de riesgo, para implementar acciones efectivas para su prevención, evitando así complicaciones innecesarias para el paciente.

Palabras clave: latrogenia, Unidad de cuidados intensivos, Atención de enfermería, Seguridad del paciente, Atención crítica.

\section{INTRODUÇÃO}

As Unidades de Terapia Intensiva (UTI) são ambientes extremamente complexos e envolvem o cuidado de pacientes em estado crítico, que necessitam de equipamentos, recursos humanos e intervenções terapêuticas especializadas. Caracteriza-se como uma unidade que demanda elevada carga de trabalho dos profissionais ali presentes, especialmente da equipe de enfermagem. Devido ao perfil dos pacientes internados e ao maior número de intervenções realizadas, o índice de ocorrência de eventos adversos neste setor é elevado (MAIA LFS e BASTIAN JC, 2013).

Os EAs podem são classificados como de natureza físico, social ou psicológica e podem comprometer estruturas ou funções do corpo e provocar efeitos nocivos, a exemplo de aparecimento de leões, incapacidades físicas com piora do quadro clínico até a morte. Estes eventos podem ser de caráter físico, social ou psicológico. Além disso, outro termo utilizado para caracterizar um evento indesejável, que causa ou tem o potencial de ocasionar resultados prejudiciais aos pacientes é iatrogenia (LIMA KP, et al., 2017; SANTANA JCB, et al., 2015).

Estimativas da OMS evidenciam que, um em cada dez pacientes sofre dano ao receber assistência hospitalar em países desenvolvidos, podendo esse número ser ainda maior em países em desenvolvimento. Diversos fatores contribuem para a ocorrência de iatrogênias, em relação ao atendimento na UTI, o que merece atenção, considerando que o paciente grave tende a estar mais susceptível a erros da equipe de enfermagem por apresentar suas particularidades (SANTANA JCB, et al., 2015).

As iatrogênias devem ser analisadas para identificar suas possíveis causas, como por exemplo, problemas estruturais, de equipamentos e também o processo de trabalho e para subsidiar medidas preventivas no ambiente hospitalar. São diversos os tipos de erros cometidos pela equipe de enfermagem, dentre eles estão: erro na administração de medicamentos, falha na execução de procedimentos, quedas, infecções, extubação acidental. Sendo estes ocasionados por falta de estrutura adequada, sobrecarga de trabalho, procedimento de alta complexidade, dimensionamento inadequado da equipe, falta de recursos necessários, desatualização dos profissionais, falta de interesse, entre outros (LIMA KP, et al., 2017; SANTANA JCB et al. 2015).

É importante ressaltar que o profissional autor e co-autor da iatrogenia estão passíveis a responder e sofrer as sanções administrativas e legais cabíveis em relação ao evento ocorrido, em especial o enfermeiro, pois este é o líder da equipe de enfermagem. Diante deste fato e com o intuito de se protegerem para não serem punidos, muitas vezes as iatrogenias não são identificadas, ocasionando assim as subnotificações e chance de o evento ocorrer novamente (DUTRA DD, et al., 2017).

Diante do contexto, este estudo objetivou identificar na produção científica brasileira, a ocorrência de iatrogenias ao paciente assistido em Unidade de Terapia Intensiva, evidenciando os fatores que propiciam os erros dos profissionais da enfermagem e discutir os cuidados de enfermagem necessários para minimização dos erros nesse cenário de cuidado.

\section{MÉTODOLOGIA}

O presente estudo trata-se de uma Revisão Integrativa da Literatura (RIL). Para guiar a presente RIL, formou-se a seguinte pergunta/questão norteadora: "Quais são os achados científicos brasileiros sobre a 
ocorrência de iatrogenias de enfermagem em UTI". Os critérios para inclusão das publicações foram: artigos completos, publicados no idioma português, na linha temporal de 2012 a 2017, com intuito de selecionar as pesquisar mais atualizadas sobre o tema, que contaram com a UTI como cenário; enquanto os critérios de exclusão: outros estudos secundários, artigos que não citassem iatrogenias provocadas pela enfermagem.

A busca de artigos foi realizada nas bases de dados Literatura Latino-Americana e do Caribe em Ciências da Saúde (LILACS), na Scientific Electronic Library Online (SCIELO) e Base de Dados em Enfermagem (BDENF), utilizando os seguintes descritores (DeCS) em ciências da saúde: latrogenia, Unidade de Terapia Intensiva, Cuidados de Enfermagem, Segurança do Paciente, Cuidados Críticos. Para estratégia de busca, foi utilizado o operador booleanõ AND. O levantamento de artigos nas bases de dados ocorreu no mês de agosto de 2018, sendo este realizado por 3 pesquisadores, em dias alternados do referido mês, nas bases de dados acima citadas, onde cada pesquisador ficou responsável pela busca em uma base de dados.

Após a localização das produções e a leitura das mesmas, foi desenvolvido um quadro que foi preenchido com as respectivas informações de cada produção científica, como o título, nome do autor, ano de publicação, objetivos e conclusão da pesquisa. A partir destas informações, realizou-se uma análise estatística descritiva para que os mesmos possam ser interpretados e quantificados a fim de obtermos o resultado da pesquisa. Assim sendo, os dados foram organizados em forma lógica, sendo estes resultados relatados sem interferências externas aos estudos utilizados, com o objetivo de conferir validade científica à pesquisa, utilizando técnicas estatísticas das mais simples as mais complexas. Posteriormente, foi realizada a discussão dos mesmos a fim de discorrer cientificamente sobre os resultados obtidos em nossa pesquisa, produzindo novos conhecimentos e respeito da temática em questão.

\section{RESULTADOS E DISCUSSÃO}

Encontraram-se um total de 112 artigos, destes, 24 publicações na base de dados BDENF, 70 na LILACS e 18 na SCIELO. Após a leitura dos mesmos, selecionou-se 14 artigos pertinentes aos objetivos do estudo. A publicação de poucos estudos a respeito de iatrogenias em Unidades de Terapia Intensiva foi uma limitação identificada na pesquisa, podendo estar relacionada à subnotificação de eventos adversos pelos profissionais, o que dificulta a abordagem do assunto. Contudo, o tema "segurança do paciente" tem sido bastante abordado atualmente, gerando interesse e conscientização por parte dos profissionais sobre a importância de evidenciar os eventos adversos ocorridos nas instituições, contribuindo deste modo para o a melhoria da qualidade da assistência prestada e gerando dados para novas pesquisar a respeito do assunto. Para interpretação dos dados e síntese, utilizou-se o quadro abaixo (Quadro 1):

A partir disso, realizou-se a análise dos conteúdos dos artigos, tomando por fundamento os objetivos desta revisão, e foram elencadas três categorias de acordo com o tema, sendo: latrogenias mais comuns na UTI, Cuidados de enfermagem na prevenção de iatrogenias, Fatores desencadeantes de latrogenias.

\section{latrogenias mais comuns na UTI}

Segundo Ortega DB, et al., (2017) assistência em saúde não está isenta de riscos e de possíveis eventos adversos. A UTI, em especial, é um ambiente onde a ocorrência destes eventos se torna mais propícia, haja vista os elementos e processos de trabalho deste setor. Diante disto, é importante o conhecimento acerca dos eventos adversos mais prevalentes em UTIs para que a partir desta informação, os profissionais tenham um cuidado e atenção redobrados na execução de sua assistência, visando à prevenção de iatrogenias e a segurança do paciente.

De acordo com Busanello J, et al., (2015), as principais iatrogênias estão associadas ao erro na administração de medicação além de procedimento invasivos e situações nos quais os pacientes estão acometidos.

Toffoleto FL, et al., (2016), afirma como o autor anterior que complicações e problemas durante a inserção de dispositivos invasivos bem como sua manutenção asséptica, inadequadas, são fatores importantes para a ocorrências das iatrogenias. 
Quadro 1 - Distribuição das publicações incluídas segundo o título, autor e ano, objetivo e conclusão.

\begin{tabular}{|c|c|c|c|}
\hline Título & Autor/Ano & Objetivo & Conclusão \\
\hline $\begin{array}{l}\text { Cuidados de enfermagem ao } \\
\text { paciente adulto: prevenção de } \\
\text { lesões cutaneomucosas e } \\
\text { segurança do paciente. }\end{array}$ & $\begin{array}{l}\text { Busanello J, } \\
\text { et al. (2015); }\end{array}$ & $\begin{array}{l}\text { Identificar os cuidados de enfermagem para } \\
\text { a prevenção das lesões cutaneomucosas em } \\
\text { pacientes adultos hospitalizados e possíveis } \\
\text { dificuldades encontradas pelos profissionais } \\
\text { para promover estas ações. }\end{array}$ & $\begin{array}{l}\text { Os cuidados realizados para a prevenção de lesões } \\
\text { cutaneomucosas em sua maioria estão de acordo } \\
\text { com o que a literatura preconiza. }\end{array}$ \\
\hline $\begin{array}{l}\text { Adesão da equipe } \text { de } \\
\text { enfermagem às medidas de } \\
\text { prevenção de infecções } \\
\text { corrente sanguínea. }\end{array}$ & $\begin{array}{l}\text { Dantas GD, } \\
\text { et al. (2017); }\end{array}$ & $\begin{array}{l}\text { Avaliar o conhecimento e adesão da equipe } \\
\text { de enfermagem às medidas de prevenção de } \\
\text { infecções de corrente sanguínea } \\
\text { relacionadas ao cateter venoso central em } \\
\text { Unidade de Terapia Intensiva. }\end{array}$ & $\begin{array}{l}\text { A adesão às medidas de prevenção de infecção da } \\
\text { corrente sanguínea deve ser incentivada através de } \\
\text { educação continuada. }\end{array}$ \\
\hline $\begin{array}{l}\text { Análise de eventos adversos } \\
\text { em pacientes internados em } \\
\text { unidade de terapia intensiva }\end{array}$ & $\begin{array}{l}\text { Ortega DB, } \\
\text { et al. (2017); }\end{array}$ & $\begin{array}{l}\text { Avaliar a incidência de eventos adversos e } \\
\text { associá-los com a carga de trabalho de } \\
\text { enfermagem, o dimensionamento da equipe } \\
\text { de enfermagem e o perfil de gravidade do } \\
\text { paciente. }\end{array}$ & $\begin{array}{l}\text { Houve maior incidência de eventos adversos em } \\
\text { pacientes que exibiram um perfil de maior risco e } \\
\text { gravidade identificados por meio de escalas } \\
\text { preditoras. }\end{array}$ \\
\hline $\begin{array}{l}\text { Fatores relacionados à } \\
\text { ocorrência de eventos } \\
\text { adversos em pacientes idosos } \\
\text { críticos. }\end{array}$ & $\begin{array}{l}\text { Toffoletto } \\
\text { MC, et al. } \\
(2016) ;\end{array}$ & $\begin{array}{l}\text { Identificar os fatores relacionados à } \\
\text { ocorrência de eventos adversos em } \\
\text { pacientes idosos críticos internados em } \\
\text { Unidade de Terapia Intensiva segundo } \\
\text { características demográficas e clínicas. }\end{array}$ & $\begin{array}{l}\text { A identificação dos eventos e fatores associados no } \\
\text { idoso subsidiam a prevenção dessas ocorrências } \\
\text { perante as vulnerabilidades dessa faixa etária. }\end{array}$ \\
\hline $\begin{array}{l}\text { Deslizes, lapsos e enganos no } \\
\text { uso de equipamentos por } \\
\text { enfermeiros na Unidade de } \\
\text { Terapia Intensiva. }\end{array}$ & $\begin{array}{l}\text { Ribeiro } \\
\text { GSR, et al. } \\
(2016)\end{array}$ & $\begin{array}{l}\text { Identificar a ocorrência de erros durante a } \\
\text { utilização dos equipamentos por enfermeiros } \\
\text { que atuam na terapia intensiva. }\end{array}$ & $\begin{array}{l}\text { Os erros causam eventos adversos que } \\
\text { comprometem a segurança do paciente. Propõe-se } \\
\text { um instrumento de verificação diária dos } \\
\text { equipamentos, com checagens ao longo do processo } \\
\text { de trabalho da programação das bombas infusoras e } \\
\text { monitores, no intuito de reduzir as falhas e } \\
\text { esquecimentos }\end{array}$ \\
\hline
\end{tabular}

REAS/EJCH | Vol.Sup.36 | e1711 | DOI: https://doi.org/10.25248/reas.e1711.2019 Página 4 de 10 


\begin{tabular}{|c|c|c|c|}
\hline $\begin{array}{l}\text { Segurança do paciente e a } \\
\text { prevenção de lesões cutâneo- } \\
\text { mucosas associadas aos } \\
\text { dispositivos invasivos nas vias } \\
\text { aéreas. }\end{array}$ & $\begin{array}{l}\text { Pinto DM, et } \\
\text { al. (2015); }\end{array}$ & $\begin{array}{l}\text { Analisar os cuidados implementados pela } \\
\text { equipe de Enfermagem para a promoção da } \\
\text { segurança do paciente adulto e a prevenção } \\
\text { de lesões cutaneomucosas associadas à } \\
\text { presença de dispositivos invasivos nas vias } \\
\text { aéreas inferiores. }\end{array}$ & $\begin{array}{l}\text { Há uma superficialidade da atuação do enfermeiro no } \\
\text { cuidado direto ao paciente e uma diferenciação em } \\
\text { relação à percepção dos técnicos de Enfermagem, } \\
\text { em especial os atuantes na unidade de terapia } \\
\text { intensiva, que apresentaram maior propriedade e } \\
\text { visão da clínica do paciente. }\end{array}$ \\
\hline $\begin{array}{l}\text { Fatores contribuintes para } \\
\text { ocorrência de eventos } \\
\text { adversos em unidade de } \\
\text { terapia intensiva: perspectiva } \\
\text { do enfermeiro. }\end{array}$ & $\begin{array}{l}\text { Lima KP, et } \\
\text { al. (2017); }\end{array}$ & $\begin{array}{l}\text { Avaliar as atitudes dos enfermeiros sobre as } \\
\text { condições que contribuem para a ocorrência } \\
\text { de eventos adversos. }\end{array}$ & $\begin{array}{l}\text { O estudo determinou as atitudes dos enfermeiros } \\
\text { frente às condições que podem favorecer a } \\
\text { ocorrência de eventos adversos através dos itens da } \\
\text { EPEA. }\end{array}$ \\
\hline $\begin{array}{l}\text { Atitude dos enfermeiros e } \\
\text { predisposição da ocorrência de } \\
\text { eventos adversos em unidade } \\
\text { de terapia intensiva. }\end{array}$ & $\begin{array}{l}\text { Lobão WM e } \\
\text { Menezes IG } \\
\text { (2017); }\end{array}$ & $\begin{array}{l}\text { Avaliar as atitudes dos enfermeiros perante } \\
\text { condições que podem predispor à ocorrência } \\
\text { de eventos adversos em UTI. }\end{array}$ & $\begin{array}{l}\text { A evidência dessa baixa percepção de forma } \\
\text { predominantemente entre especialistas aponta para } \\
\text { a necessidade de inclusão nos currículos de } \\
\text { graduação em Enfermagem e pós-graduação em } \\
\text { terapia intensiva da discussão sobre eventos } \\
\text { adversos e a qualidade do cuidado de enfermagem. }\end{array}$ \\
\hline $\begin{array}{l}\text { Higienização das mãos: } \\
\text { adesão da equipe de } \\
\text { enfermagem de unidades de } \\
\text { terapia intensiva pediátricas. }\end{array}$ & $\begin{array}{l}\text { Raimondi } \\
\text { DC, et al. } \\
(2017) \text {; }\end{array}$ & $\begin{array}{l}\text { Investigar e confrontar a adesão da equipe } \\
\text { de enfermagem de UTI de três hospitais } \\
\text { quando a higienização das mãos. }\end{array}$ & $\begin{array}{l}\text { A adesão das equipes investigadas apresentou-se } \\
\text { insatisfatória à higienização das mãos e; profissionais } \\
\text { técnicos de enfermagem se mostraram menos } \\
\text { aderentes que enfermeiros. }\end{array}$ \\
\hline $\begin{array}{l}\text { Uso de tecnologias na terapia } \\
\text { intravenosa: contribuições para } \\
\text { uma prática mais segura. }\end{array}$ & $\begin{array}{l}\text { Moreira } \\
\text { APA, et al. } \\
(2017) ;\end{array}$ & $\begin{array}{l}\text { Identificar quais são as dificuldades da } \\
\text { equipe de Enfermagem no manejo das } \\
\text { tecnologias durante a terapia intravenosa } \\
\text { (TIV) e discutir as dificuldades identificadas } \\
\text { sob a perspectiva da segurança do paciente }\end{array}$ & $\begin{array}{l}\text { A falta de treinamento é apresentada como a maior } \\
\text { difi culdade dos profi ssionais de Enfermagem e } \\
\text { permeia as questões de segurança do paciente e do } \\
\text { profissional ao utilizar as tecnologias duras na TIV }\end{array}$ \\
\hline $\begin{array}{l}\text { Segurança do paciente } \mathrm{e} \\
\text { enfermagem: interface rom } \\
\text { estresse e síndrome de } \\
\text { Burnout. }\end{array}$ & $\begin{array}{l}\text { Rodrigues } \\
\text { CCFM, et al. } \\
\text { (2017); }\end{array}$ & $\begin{array}{l}\text { Analisar estudos que versam sobre o } \\
\text { estresse e Síndrome de Burnout, bem como } \\
\text { a segurança do paciente no âmbito da } \\
\text { assistência de enfermagem no ambiente } \\
\text { hospitalar. }\end{array}$ & $\begin{array}{l}\text { A análise apontou que o estresse e a Síndrome de } \\
\text { Burnout vivenciada por esses profissionais acarretam } \\
\text { maior vulnerabilidade ao desenvolvimento de uma } \\
\text { assistência insegura e que fatores como a falta de } \\
\text { apoio organizacional podem contribuir para dirimir } \\
\text { essas falhas. }\end{array}$ \\
\hline
\end{tabular}

REAS/EJCH | Vol.Sup.36 | e1711 | DOI: https://doi.org/10.25248/reas.e1711.2019 Página 5 de 10 


\begin{tabular}{|c|c|c|c|}
\hline $\begin{array}{l}\text { Cultura de segurança do } \\
\text { paciente em unidade de terapia } \\
\text { intensiva: perspectiva da } \\
\text { equipe de enfermagem. }\end{array}$ & $\begin{array}{l}\text { Mello JF e } \\
\text { Barbosa } \\
\text { SFF (2017); }\end{array}$ & $\begin{array}{l}\text { Identificar e comparar as dimensões da } \\
\text { cultura de segurança do paciente na } \\
\text { perspectiva dos profissionais de } \\
\text { enfermagem de duas Unidades de Terapia } \\
\text { Intensiva. }\end{array}$ & $\begin{array}{l}\text { A cultura de segurança precisa ser desenvolvida nos } \\
\text { locais do estudo, com especial atenção aquelas com } \\
\text { avaliação menos positiva sobre a cultura de } \\
\text { segurança }\end{array}$ \\
\hline $\begin{array}{l}\text { Eventos adversos e segurança } \\
\text { na assistência de enfermagem. }\end{array}$ & $\begin{array}{l}\text { Duarte } \\
\text { SCM, et al. } \\
(2015)\end{array}$ & $\begin{array}{l}\text { ldentificar as publicações científicas sobre os } \\
\text { eventos adversos na assistência de } \\
\text { enfermagem em pacientes adultos } \\
\text { hospitalizados e discutir os principais } \\
\text { eventos adversos na assistência de } \\
\text { enfermagem. }\end{array}$ & $\begin{array}{l}\text { É importante discutir estratégias de prevenção de } \\
\text { eventos realmente eficazes, que assegurem a } \\
\text { segurança do paciente nas instituições de saúde. }\end{array}$ \\
\hline $\begin{array}{l}\text { Carga de trabalho da equipe de } \\
\text { enfermagem e segurança do } \\
\text { paciente - estudo com método } \\
\text { misto na abordagem ecológica } \\
\text { restaurativa. }\end{array}$ & $\begin{array}{l}\text { Magalhães } \\
\text { AMM, et al. } \\
(2013) \text {; }\end{array}$ & $\begin{array}{l}\text { Analisar a carga de trabalho da equipe de } \\
\text { enfermagem e sua potencial relação com a } \\
\text { segurança do paciente, em unidades de } \\
\text { internação das áreas clínica e cirúrgica de } \\
\text { um hospital universitário. }\end{array}$ & $\begin{array}{l}\text { Os achados deste estudo evidenciaram associações } \\
\text { significativas entre carga de trabalho e a segurança } \\
\text { dos pacientes. Observa-se que os quadros de } \\
\text { pessoal com menos pacientes apresentaram os } \\
\text { melhores indicadores de qualidade assistencial e } \\
\text { gerencial de segurança do paciente. }\end{array}$ \\
\hline
\end{tabular}

Fonte: Carmo BKO, Santos BP, Nascimento CL, et al., 2019. 
Além disso, Ortega DB, et al., (2017) destacou a ocorrência de iatrogenias como a perda da sonda nasoenteral e o desenvolvimento de lesões por pressão. Onde a maior incidência de eventos adversos foi em pacientes com idade mais elevada, maior tempo de internação, além de escores de maior gravidade clínica, maior risco para lesão por pressão e maior carga de trabalho.

Foram encontradas ainda, evidências relacionadas à utilização dos instrumentos utilizados no cuidado ao paciente na UTI, tais como as bombas infusoras (BI) e sistemas de monitorização, com isso observam-se inúmeras situações de erros que comprometem a segurança do paciente, tais como lapsos, pequenos deslizes e/ou confusão dos profissionais enfermeiros durante a utilização de tais equipamentos na UTI (RIBEIRO GSR, et al., 2016).

Dentre os eventos envolvendo as $\mathrm{BI}$ destacam-se falta de atenção aos alarmes disparados pelos equipamentos, os erros na execução da programação da máquina durante o manuseio, não só na administração de medicação quanto da dieta, o que pode acarretar eventos adversos necessitando ou não de intervenção para a manutenção da vida. Tais erros podem ser justificados pelo ato da não utilização dos padrões adequados à realidade de cada paciente e pela falta de conhecimento exigido para manipulação da máquina (RIBEIRO GSR, et al., 2016).

Frente à utilização de dispositivos invasivos nas vias aéreas, evidenciam-se lesões cutâneo-mucosas que acometem os pacientes tanto nas vias aéreas superiores e inferiores. Percebe-se que a permanência do uso destes dispositivos bem como sua inadequada manutenção e higienização são pequenas falhas que podem acarretar danos irreparáveis ao paciente, caracterizados como traqueomalacia, estenose traqueal, granuloma, fissuras orais, paralisia e parestesia das cordas vocais úlceras laríngeas e nas cordas vocais, principalmente em pacientes que fazem uso da traqueostomia. Devido ao tempo excessivo, porém, necessário do dispositivo nas vias áreas e as danosas extubações acidentais que frequentemente acontecem em banho no leito, mudança de decúbito, transporte do paciente e troca da fixação, as vias aéreas desses pacientes são lesionadas (PINTO DM, et al., 2015).

Sendo assim, percebe-se que a ocorrência de eventos adversos quanto ao uso de dispositivos e equipamentos podem estar relacionados à complexidade e variedade dos dispositivos, de seus fabricantes, suas especificações e técnicas de funcionamento de cada equipamento. Diante do que pode ocorrer em uma unidade de terapia intensiva, os profissionais, em especial, a equipe de enfermagem deve possuir competência e habilidades para garantir assistência segura, eficiente e eficaz a esses pacientes (PINTO DM, et al., 2015).

\section{Cuidados de enfermagem na prevenção de iatrogenias}

A segurança do paciente é definida pela Organização Mundial de Saúde (OMS) como a redução do risco de dano desnecessário relacionado à assistência em saúde, diminuição dos atos inseguros no cuidado ao paciente e adoção de melhores práticas assistenciais em vista de alcançar os melhores resultados possíveis para o tratamento deste (LIMA KP, et al., 2017).

A equipe de enfermagem tem grande importância no contexto da segurança do paciente para prevenir incidentes e agravos, pois estes são os profissionais mais próximos no cuidado diário ao paciente (BUSANELLO J, et al., 2015).

Segundo Lobão WM e Menezes IG (2017), a qualidade do cuidado de Enfermagem em Unidades de Terapia Intensiva parte da necessidade de se garantir a segurança do paciente, visto que a ocorrência de um evento adverso interfere negativamente sobre o resultado esperado, podendo comprometer a vida do paciente, seu tempo de permanência no hospital, a confiança na equipe e o aumento dos gastos relativos ao seu cuidado.

Portanto, o cuidado de enfermagem é o resultado das condições de trabalho impostas, o que envolve estrutura e processo, por recomendações nacionais e internacionais da promoção da segurança do paciente em UTI, e as atitudes dos enfermeiros intensivistas sobre as condições que podem predispor à ocorrência do evento adverso (LIMA KP, et al., 2017). 
Neste contexto, sabe-se que durante a prestação do cuidado as mãos dos profissionais da saúde são contaminadas por agentes patógenos, constituindo-se no principal mecanismo de transmissão de microrganismos de um local para outro, de um paciente para outro ou de um local contaminado para os pacientes. Deste modo, a adesão ao procedimento de higienização das mãos de forma rotineira é constantemente associada a práticas seguras do cuidado à saúde, em especial em setores de alta complexidade destinados à pacientes considerados vulneráveis, que é o caso da UTI. Sendo assim, é primordial a prática de higienização das mãos pelos profissionais da área da saúde a fim de evitar e reduzir as infecções associadas aos cuidados de saúde (RAIMONDI DC, et al., 2017).

Além disso, em um estudo de Busanello J, et al., (2015) os profissionais de enfermagem citaram medidas de prevenção para úlcera por pressão como, mudança de decúbito a cada duas horas; hidratação da pele, com o uso de hidratantes ou óleo mineral; higiene corporal; massagem de conforto; e proteção das proeminências ósseas, além da importância dos cuidados para a prevenção de lesões orais, destacando como imprescindível a higiene com o uso de antisséptico bucal e a hidratação dos lábios com óleo mineral. As lesões relacionadas ao uso de sonda para gavagem estão: a alternância das narinas na troca da sonda; a troca da fixação desta no momento da higiene corporal do paciente, utilizando fita micropore ou esparadrapo e alternando os locais de fixação; quanto ao cateter nasal, é fundamental manter a concentração de oxigênio em fluxo adequado para evitar lesões na mucosa nasal (BUSANELLO J, et al., 2015).

Em relação ao acessovenoso periférico, a troca do cateter venoso dentro do prazo estabelecido pela OMS, o uso de micropore em pessoas idosas, a observação desinais de flebite e fluidez do acesso, e realização daassepsia da pele com álcool $70 \%$, são cuidados essenciais para evitar lesões. Outro dispositivo que demanda pratica asséptica são as sondas vesicais principalmente as de demora (BUSANELLO J, et al., 2015).

Pode-se destacar ainda o papel do enfermeiro na prevenção de erros e na garantia de maior segurança na administração de medicamentos, principalmente via intravenosa, pois isto requer muita atenção em virtude do grande volume de fármacos necessários ao paciente internado em uma UTI, o que implica na necessidade de normas de preparo e administração mais severas em vistas as possibilidades de complicações advindas de más pratica associadas. Bem como que o planejamento da assistência de enfermagem deve viabilizar medidas de prevenção de iatrogênias na prescrição de enfermagem (MOREIRA APA, et al., 2017; PINTO DM, 2015).

Portanto, Raimondi DC, et al., (2017) enfatiza a instituição de protocolos de prevenção de eventos adversos como sendonecessária para a prevenção de iatrogenias, porém não sendo a única medida que consegue obter impactos na redução da incidência e na ocorrência de complicações. É necessário também que os profissionais tenham conhecimento e possuam alta adesão às diretrizes recomendadas, bem como, deve-se avaliar o conhecimento e as práticas dos profissionais de saúde no cenário das UTIs, o que justifica a necessidade de pesquisas que investiguem o conhecimento e adesão dos profissionais da saúde às medidas de prevenção de iatrogenias neste cenário de atuação.

Os esforços para a prevenção de eventos adversos devem visar à qualidade da assistência e segurança do paciente, minimizando a ocorrência destes por meio da identificação e implementação de estratégias interventivas que visem zerar a ocorrência dessa complicação em pacientes críticos na UTI (DANTAS GD, et al., 2017).

\section{Fatores desencadeantes de latrogenias}

A ocorrência de eventos adversos está comumente associada a erros individuais, mas deve-se ressaltar os fatores que os desencadeiam, como as condições de trabalho oferecidas e a complexidade das ações realizadas. $O$ avanço tecnológico aliado ao déficit no aperfeiçoamento dos recursos humanos, a falha na Sistematização da Assistência de Enfermagem (SAE) e sobrecarga com muitas horas de trabalho predispõem ao risco da ocorrência de eventos adversos (LIMA KP, et al., 2017).

De acordo com Rodrigues CCFM, et al., (2017), os fatores humanos estão vinculados a questões institucionais, ambientais, fisiológicos, psicológicos, além do conhecimento e habilidades visto o despreparo para a qualificação profissional. Este autor também menciona sobre a insatisfação com o trabalho, lesões 
ocupacionais e violência contra o profissional que afetam a qualidade da pratica de enfermagem e segurança do paciente.

O quantitativo de pessoal é um importante indicador de segurança do paciente. Pacientes assistidos por profissionais sobrecarregados têm mais do que o dobro de chance de sofrerem ao menos um evento adverso. As complicações resultantes da sobrecarga de trabalho do enfermeiro aumentaram o tempo de internação e o risco de mortalidade dos pacientes (MELO JF e BARBOSA SFF, 2017).

Além disso, os achados de associação significativa entre as cargas de trabalho da equipe de enfermagem e eventos adversos, como quedas do leito e infecções relacionadas ao cateter venoso central demonstraram que, dentro do ambiente estudado, o aumento do número de pacientes designados para cada enfermeiro, ou auxiliar/técnico de enfermagem, aumenta a incidência desses indicadores, tendo impacto negativo na segurança do paciente. Ou seja, o dimensionamento adequado do pessoal de enfermagem com menores taxas de paciente/profissional, ajudam a reduzir a incidência de eventos adversos, incluindo quedas do leito e infecções (MAGALHÃES AMM, et al., 2013).

Dantas GD, et al., (2017) salienta-se também que a falta de conhecimento é um dos fatores que mais contribuem para a ocorrência de eventos adversos. Esse fenômeno pode repercutir em danos aos pacientes e, ainda, em prejuízos aos profissionais devido aos aspectos éticos e legais relacionados.

As reações dos profissionais frente aos eventos adversos da equipe de enfermagem denotam que, o erro não é intencional, e muitas vezes, o profissional não percebe a ocorrência. Outro fator é o reconhecimento dos profissionais da impossibilidade de manter sempre a atenção prestada na atividade executada, descartando a intencionalidade direcionada para o erro. O reconhecimento dos eventos adversos e demais ocorrências também podem ser relacionado à cultura, crença e conhecimento dos profissionais acerca do problema, uma vez que alguns profissionais apresentam dificuldades em perceber o erro (DUARTE SCM, et al., 2015).

Os fatores para a ocorrência de eventos adversos estão relacionados a carga de trabalho, o dimensionamento do pessoal bem como, o relacionamento interpessoal pela equipe multiprofissional e mau gerenciamento causado pela falta de liderança (DUARTE SCM, et al., 2015).

Moreira IA, et al., (2015) ressalta que os procedimentos realizados nas instituições de saúde, normalmente envolvem determinados riscos que são agravados na presença de falhas na estrutura e processos da assistência, tornando o cuidado inseguro e com baixa qualidade. Revela também que o entendimento dos profissionais sobre os eventos adversos é superficial, evidenciando a necessidade de políticas gerenciais, que visem educação na saúde.

Outro ponto crucial é a falta comunicação entre os profissionais correta da ocorrência de eventos adversos, pois, a maioria relata o ocorrido, verbalmente, ao enfermeiro ou ao médico responsáveis pelo setor. Estes profissionais que apresentam esta conduta documentam o fato ocorrido em prontuários blocos de anotações de enfermagem, porém não expõem o evento adverso em notificações sistemáticas que facilitam a análise geral dos serviços prestados, dentre eles a ocorrência de eventos adversos (MOREIRA IA, et al., 2015).

Sendo assim, evidencia-se que a ocorrência de iatrogênia apresenta uma esfera multifacetada, os riscos existem naturalmente por serem procedimentos invasivos. Porém há fatores que colaboram para a existência do fato, dentre eles, a falta de estrutura dos setores e equipamentos, grande jornada de trabalho, profissionais insatisfeitos com o reconhecimento financeiro e moral de seus serviços prestados, bem como falta de liderança e supervisão de enfermagem adequadas (DUARTE SCM, et al., 2015).

\section{CONCLUUSÃO}

Diante dos estudos analisados, conclui-se que a ocorrência de iatrogenias é uma realidade nas Unidades de Terapia Intensiva, possuindo um elevado risco de acontecer devido à complexidade do setor e dos procedimentos realizados. Está relacionada a carga de trabalho elevada, estrutura que não supre as necessidades dos profissionais, relação da equipe multiprofissional, atos repetitivos e mecanicistas, 
dificuldade na adesão ao protocolo de segurança do paciente, dimensionamento, bem como falta de conhecimento. Foi possível constatar a importância do enfermeiro na prevenção de eventos adversos, pois ele é o responsável pela gerência da assistência e do cuidado, por isso deve ter suas ações pautadas no planejamento e organização adequada do ambiente de trabalho, o que envolve recursos materiais e humanos, para promover uma assistência de enfermagem de qualidade reduzindo cada vez mais os eventos adversos ao seu paciente.

\section{REFERÊNCIAS}

1. BUSANELLO J, et al. Cuidados de enfermagem ao paciente adulto: prevenção de lesões cutaneomucosas e segurança do paciente. Revista de Enfermagem da Universidade Federal de Santa Maria, 2015; 5(4):597-606.

2. DANTAS GD, et al. Adesão da equipe de enfermagem às medidas de prevenção de infecções de corrente sanguínea. Revista Enfermagem UFPE online, 2017; 11(10):3698-3706.

3. DUARTE SCM, et al. Eventos adversos e segurança na assistência de enfermagem. Revista Brasileira de Enfermagem, 2015; 68 (1): 144-154.

4. DUTRA DD, et al. Eventos adversos em Unidades de Terapia Intensiva: estudo bibliométrico. RevFundCare Online. 2017 jul/set; 9(3):669-675.

5. LIMA KP, et al. Fatores contribuintes para ocorrência de eventos adversos em unidade de terapia intensiva: perspectiva do enfermeiro. Revista Enfermagem UFPE online, 2017; 11(3):1234-1243.

6. LOBÃO WM, MENEZES IG. Atitude dos enfermeiros e predisposição da ocorrência de eventos adversos em unidade de terapia intensiva. Revista Enfermagem UFPE online, 2017; 11(5):1971-1979.

7. MAIA LFS, BASTIAN JC. latrogenias: ações do enfermeiro na prevenção de ocorrências iatrogênicas em unidade de terapia intensiva. Revista Recien, 2013; 3(7):27-35.

8. MAGALHÃES AMM, et al. Carga de trabalho da equipe de enfermagem e segurança do paciente - estudo com método misto na abordagem ecológica restaurativa. Rev. Latino-Am. Enfermagem. Jan.-fev. 2013; 21(Spec): [09 telas].

9. MELLO JF, BARBOSA SFF. Cultura de segurança do paciente em unidade de terapia intensiva: perspectiva da equipe de enfermagem. Revista Eletrônica Enfermagem, 2017; 19(7):2-12.

10. MOREIRA APA, et al. Uso de tecnologias na terapia intravenosa: contribuições para uma prática mais segura. Revista Brasileira de Enfermagem, 2017; 70(3):623-629.

11. MOREIRA IA, et al. Conhecimento dos profissionais de saúde sobre eventos adversos em unidade de terapia intensiva. Revista de enfermagem UERJ, 2015; 23(4):461-467.

12. ORTEGA DB, et al. Análise de eventos adversos em pacientes internados em unidade de terapia intensiva. Acta Paulista de Enfermagem,2017; 30(2):168-173.

13. PINTO DM, et al. Segurança do paciente e a prevenção de lesões cutâneo-mucosas associadas aos dispositivos invasivos nas vias aéreas. Revista da Escola de Enfermagem da USP,2015; 49(5):775-782.

14. RAIMONDI DC, et al. Higienização das mãos: adesão da equipe de enfermagem de unidades de terapia intensiva pediátricas. Revista Cuidarte, 2017; 8(3):1839-48.

15. RIBEIRO GSR, et al. Deslizes, lapsos e enganos no uso de equipamentos por enfermeiros na Unidade de Terapia Intensiva. Revista da Escola de Enfermagem da USP, 2016; 50(3):419-426.

16. RODRIGUES CCFM, et al. Segurança do paciente e enfermagem: interface com estresse e síndrome de Burnout. Revista Brasileira de Enfermagem,2017; 70(5): 1141-1147.

17. SANTANA JCB, et al. latrogenias na assistência em uma unidade de terapia intensiva: percepção da equipe de enfermagem. Enfermagem revista, 2015;18(02):3-17.

18. TOFFOLETTO MC, et al. Fatores relacionados à ocorrência de eventos adversos em pacientes idosos críticos. Revisa Brasileira de Enfermagem, 2016;69(6):1039-1045. 